\title{
Spiral Gradient Dilution, a Rapid Method for Determining Growth Responses and 50\% Effective Concentration Values in Fungus-Fungicide Interactions
}

\author{
Helga Förster, Loukas Kanetis, and James E. Adaskaveg
}

Department of Plant Pathology, University of California, Riverside 92521.

Accepted for publication 21 September 2003.

\begin{abstract}
Förster, H., Kanetis, L., and Adaskaveg, J. E. 2004. Spiral gradient dilution, a rapid method for determining growth responses and $50 \%$ effective concentration values in fungus-fungicide interactions. Phytopathology 94:163-170.

A new technique, the spiral gradient dilution method, was evaluated for determining $50 \%$ effective concentrations $\left(\mathrm{EC}_{50}\right.$ values) of fungicides for the inhibition of mycelial growth and conidial germination of various fungi. In this method, an agar medium is plated with a fungicide solution by means of a spiral plater, which applies the fungicide in a $2.5-\log$ dilution in a continuous radial concentration gradient. Fungal inoculum is then placed along radial lines across the gradient. After incubation of the plates, distinct growth shapes were observed in different fungusfungicide interactions. Mycelial growth responses to increasing fungicide concentrations ranged from abrupt to gradual transitions. Conidial germination responses were similar; in addition, distinct zones of confluent growth, nonconfluent growth, and outlier colonies were also identified, depending on the fungus-fungicide interaction. The fungicide concentra-

media, was calculated by a computer program. $\mathrm{EC}_{50}$ values were obtained for mycelial growth in 22 fungus-fungicide interactions and for conidial germination in five interactions. The fungi evaluated were members of the Zygomycota, Ascomycota, Basidiomycota, and Deuteromycota. Nine fungicides, belonging to six different chemical classes, were tested. $\mathrm{EC}_{50}$ values were compared with those obtained by the traditional agar dilution method. In linear regression analyses of the two methods, the models were highly significant $(P<0.01)$, and coefficients of determination $\left(r^{2}\right)$ were 0.92 for the mycelial growth assays and 0.94 for the conidial germination assays. Regression slopes were not significantly different from $1(P>0.05)$ with optimal program settings in the software. Estimated bias, coefficients of variation, and actual confidence intervals for the regression slope were $13.5 \%, 6.5 \%$, and $1.14 \pm 0.14$ for the mycelial growth assays and $7.5 \%, 14.5 \%$, and $1.08 \pm 0.37$ for the conidial germination assays. These analyses indicate that the spiral gradient dilution method is accurate and precise compared with the agar dilution method for calculating $\mathrm{EC}_{50}$ values of fungicides in continuous growth responses to fungicide concentration gradients.
\end{abstract} tion at the radial distance at which $50 \%$ reduction of growth or spore germination occurred, compared with growth or germination on unamended
Additional keyword: fungicide toxicity.
The degree of toxicity of a fungicide to a target organism in vitro is generally expressed as the $50 \%$ effective concentration $\left(\mathrm{EC}_{50}\right)$, i.e., the fungicide concentration at which mycelial growth or spore germination of the target fungus is reduced by $50 \%$. Traditionally, $\mathrm{EC}_{50}$ values are determined by serial dilution of a fungicide in an agar medium or by a bioassay $(3,4)$. Both of these methods are time-, labor-, and resource-intensive. The agar dilution method requires the preparation of large amounts of agar media and several fungicide stock solutions, pouring replicate plates for each fungicide concentration, inoculating the plates, and measuring fungal growth after incubation. A recently described microassay in which fungal growth is optically measured in microtiter plates (6) affords considerable savings in supplies, but this technique still involves pipetting at many steps and requires special equipment (e.g., a microtiter plate reader). In addition, this assay is of limited use for analysis of highly hydrophobic compounds, because precipitation interferes with the measurement of optical density (6). For bioassays, uniform plant tissues have to be available. They are treated with fungicides at selected concentrations, inoculated with the test fungus, and evaluated for disease $(8,10)$. The selection of appropriate fungicide concentrations is difficult in both methods, because growth inhibition or disease reduction values should ideally be distributed over a

Corresponding author: J. E. Adaskaveg; E-mail address: jim.adaskaveg@ucr.edu

Publication no. P-2003-1113-02R

(c) 2004 The American Phytopathological Society wide range. $\mathrm{EC}_{50}$ values from dilution plating studies are obtained by regression analysis of probit- or logit-transformed growth inhibition data on the logarithm of the fungicide concentration (4).

There is a need for a more rapid and cost-effective method, because the quantification of fungicide toxicity is vital in a range of applications. $\mathrm{EC}_{50}$ values are used in selecting the most effective compounds in fungicide screenings and to quantitatively compare the efficacy of different compounds in vitro. In fungicide resistance management, baseline sensitivities have become critical parameters that define the distribution of $\mathrm{EC}_{50}$ values in a fungal population that has not been previously exposed to a particular fungicide $(7,10,18)$. After the introduction of the fungicide, fungal populations are periodically monitored to detect the development of insensitivity at an early stage $(8,14)$. Large numbers of isolates have to be evaluated during this monitoring to obtain an accurate assessment of fungicide sensitivity.

The spiral gradient endpoint method for determining minimal inhibitory concentrations of antibacterial agents was introduced in 1990 and has been extensively used in clinical studies of bacteria (12). In this method, a precise volume of a stock solution of a test substance is plated in an Archimedes spiral on a rotating agar plate, from the center to the perimeter, by means of a spiral plater, forming a $2.5-\log$ dilution in a radial concentration gradient. Test bacteria are applied along radial lines, and the radial location of the transition from growth to reduced growth is measured. The minimum inhibitory concentration is computed from the measured radial location by the Spiral Gradient Endpoint (SGE) software 
(Spiral Biotech, Norwood, MA). The spiral gradient endpoint method has been compared with the agar dilution method in tests with a wide range of bacteria $(2,16,17)$. Although found to be very accurate and reproducible, the spiral gradient endpoint method has never been used with fungi. Furthermore, because minimum inhibitory concentration is the most common measure of toxicity in bacterial studies, $\mathrm{EC}_{50}$ values have not previously been determined by this method. Thus, the objective of this study was to evaluate the spiral gradient endpoint method for determining fungicide toxicity and to adapt the method to determine $\mathrm{EC}_{50}$ values. The accuracy and precision of the method was assessed by comparison to $\mathrm{EC}_{50}$ values obtained by the traditional agar dilution method.

\section{MATERIALS AND METHODS}

Fungal isolates, culturing, and inoculum preparation. The fungal isolates evaluated are listed in Table 1. For the mycelial growth assays, the fungi were cultured on potato dextrose agar (PDA) (Difco Laboratories, Detroit), except for Penicillium digitatum, which was grown on cornmeal agar (Difco) for the agar dilution method, to reduce sporulation in the production of mycelial inoculum. For conidial production, Botrytis cinerea was grown on King's Medium B (1) under fluorescent light (type 42T12, Philips, New York) at approximately 600 lux. Monilinia fructicola and $P$. digitatum were grown in the dark on clarified V8 juice agar (13) and PDA, respectively. To produce mycelial inoculum for the spiral gradient dilution method, the fungi were grown on hydrophilic cellophane (du Pont de Nemours, Wilmington, DE) that had been cut into strips $(50 \times 3$ to $4 \mathrm{~mm})$ and sterilized by autoclaving in distilled water. Six or seven strips were then placed on PDA in a $10-\mathrm{cm}$ petri dish. A conidial suspension of the test fungus $\left(100 \mu\right.$ of $5 \times 10^{5}$ to $1 \times 10^{7}$ conidia per $\mathrm{ml}$ ) was spread evenly over the strips and the agar. The strips were then incubated at $25^{\circ} \mathrm{C}$ for 1 or 2 days and were used before fungal sporulation occurred. For Chondrostereum purpureum, a fungus that does not readily produce spores in culture (9), mycelial agar strips were placed between the cellophane strips, and the plates were incubated at $25^{\circ} \mathrm{C}$ for 3 or 4 days.

Fungicides evaluated. The fungicides evaluated with each fungal isolate are listed in Table 1. All fungicides used were formulated products in aqueous suspensions. The fungicides included benomyl (Benlate 50WP) (du Pont de Nemours, Wilmington, DE), a benzimidazole; dicloran (Allisan 75WP) (Gowan Co., Yuma, AZ), an aromatic hydrocarbon; fenhexamid (Elevate 50WG) (Arvesta, San Francisco), a hydroxyanilide; fludioxonil (Scholar 50WP) (Syngenta Crop Protection, Greensboro, NC), a phenylpyrrole; and iminoctadine tris-albesilate (Bellkute 30FL) (Dainippon Ink \& Chemicals, Tokyo), a guanidine. Also included were four sterol biosynthesis inhibitors: myclobutanil, as Rally 40WP (Dow AgroSciences LLC, Indianapolis) for tests with Colletotrichum spp. and as Laredo 2EC (Dow AgroSciences) for tests with Chondrostereum purpureum; propiconazole (Break 3.6EC) (Syngenta Crop Protection); tebuconazole (Elite 45WP) (Bayer CropScience, Kansas City, MO); and tetraconazole, as Eminent 125SL (Sipcam Agro USA, Inc., Roswell, GA) or TM-415 (Arvesta). Both formulations of tetraconazole were used for $P$. digitatum. The molecular weights used for the calculation of stock concentrations were, for benomyl, 290; dicloran, 207; fenhexamid, 302; fluidoxonil, 248; iminoctadine tris-albesilate, 1,335; myclobutanil, 289; propiconazole, 342; tebuconazole, 308; and tetraconazole, 372.

Agar dilution method. Molten PDA was cooled to approximately $50^{\circ} \mathrm{C}$, and stock solutions of fungicides in sterile distilled water were added. Final concentrations were adjusted so that inhibition values for mycelial growth and conidial germination were evenly distributed over a wide range of concentrations and included values above and below 50\% inhibition.

In the mycelial growth assays, mycelial agar plugs from the edge of a culture of each fungal isolate (1-day-old cultures of Rhizopus stolonifer and 5- to 7-day-old cultures of the other fungi) were placed in the centers of three or four replicate plates for each fungicide concentration. Radial growth was measured

TABLE 1. Values for $50 \%$ effective concentration $\left(\mathrm{EC}_{50}\right)$ for inhibition of mycelial growth in selected fungus-fungicide combinations as determined by the agar dilution method and the spiral gradient dilution (SGD) method

\begin{tabular}{|c|c|c|c|c|c|c|c|}
\hline \multirow[b]{2}{*}{ Fungicide } & \multirow[b]{2}{*}{ Fungus } & \multirow[b]{2}{*}{ Isolate no. } & \multicolumn{5}{|c|}{$\mathrm{EC}_{50}$ for inhibition of mycelial growth (mg/liter) ${ }^{\mathrm{a}}$} \\
\hline & & & Agar dilution & SGD 2-dayb & Std. error & SGD 1-dayb & Std. error \\
\hline Benomyl & Colletotrichum gloeosporioides & 8 & 0.091 & 0.125 & 0.014 & 0.202 & 0.032 \\
\hline Dicloran & $\begin{array}{l}\text { Botrytis cinerea } \\
\text { Monilinia fructicola }\end{array}$ & $\begin{array}{r}1356 \\
90\end{array}$ & $\begin{array}{l}2.310 \\
1.482\end{array}$ & $\begin{array}{l}3.088 \\
2.059\end{array}$ & $\begin{array}{l}0.355 \\
0.178\end{array}$ & $\begin{array}{l}5.363 \\
2.318\end{array}$ & $\begin{array}{l}0.755 \\
0.748\end{array}$ \\
\hline Fenhexamid & M. fructicola & 90 & 0.047 & 0.033 & 0.006 & 0.052 & 0.010 \\
\hline Fludioxonil & $\begin{array}{l}\text { B. cinerea } \\
\text { M. fructicola } \\
\text { Rhizopus stolonifer }\end{array}$ & $\begin{array}{r}1356 \\
90 \\
1535\end{array}$ & $\begin{array}{l}0.050 \\
0.010 \\
0.027\end{array}$ & $\begin{array}{l}0.025 \\
0.008 \\
0.047\end{array}$ & $\begin{array}{l}0.000 \\
0.001 \\
0.000\end{array}$ & $\begin{array}{l}0.040 \\
0.013 \\
0.079\end{array}$ & $\begin{array}{l}0.001 \\
0.002 \\
0.001\end{array}$ \\
\hline $\begin{array}{l}\text { Iminoctadine } \\
\text { tris-albesilate }\end{array}$ & $\begin{array}{l}\text { Geotrichum citri-aurantii } \\
\text { Penicillium digitatum }\end{array}$ & $\begin{array}{l}1741 \\
1726\end{array}$ & $\begin{array}{l}0.040 \\
0.013\end{array}$ & $\begin{array}{l}0.030 \\
0.012\end{array}$ & $\begin{array}{l}0.003 \\
0.005\end{array}$ & $\begin{array}{l}0.035 \\
0.016\end{array}$ & $\begin{array}{l}0.005 \\
0.008\end{array}$ \\
\hline Myclobutanil & $\begin{array}{l}\text { Chondrostereum purpureum } \\
\text { C. purpureum } \\
\text { C. purpureum } \\
\text { Colletotrichum acutatum } \\
\text { C. gloeosporioides }\end{array}$ & $\begin{array}{r}2249 \\
2303 \\
2306 \\
1772 \\
8\end{array}$ & $\begin{array}{l}0.145 \\
0.230 \\
0.151 \\
1.740 \\
3.445\end{array}$ & $\begin{array}{l}0.150 \\
0.280 \\
0.169 \\
1.845 \\
3.684\end{array}$ & $\begin{array}{l}0.032 \\
0.030 \\
0.066 \\
0.331 \\
0.253\end{array}$ & $\begin{array}{l}0.263 \\
0.450 \\
0.268 \\
2.972 \\
5.925\end{array}$ & $\begin{array}{l}0.05 \\
0.059 \\
0.114 \\
0.747 \\
0.576\end{array}$ \\
\hline Propiconazole & C. acutatum & 1732 & 0.550 & 0.247 & 0.072 & 0.382 & 0.128 \\
\hline Tebuconazole & $\begin{array}{l}\text { B. cinerea } \\
\text { C. acutatum } \\
\text { G. citri-aurantii } \\
\text { M. fructicola } \\
P . \text { digitatum } \\
\text { R. stolonifer }\end{array}$ & $\begin{array}{r}1356 \\
1732 \\
1741 \\
90 \\
1726 \\
1535\end{array}$ & $\begin{array}{l}0.126 \\
0.330 \\
2.230 \\
0.001 \\
0.063 \\
1.840\end{array}$ & $\begin{array}{l}0.131 \\
0.129 \\
1.631 \\
0.009 \\
0.115 \\
2.980\end{array}$ & $\begin{array}{l}0.007 \\
0.028 \\
0.257 \\
0.003 \\
0.037 \\
0.070\end{array}$ & $\begin{array}{l}0.208 \\
0.206 \\
2.585 \\
0.014 \\
0.193 \\
4.397\end{array}$ & $\begin{array}{l}0.016 \\
0.054 \\
0.577 \\
0.005 \\
0.065 \\
0.613\end{array}$ \\
\hline Tetraconazole & P. digitatum & 1726 & 0.150 & 0.312 & 0.102 & 0.473 & 0.178 \\
\hline
\end{tabular}

${ }^{a} \mathrm{EC}_{50}$ is the effective concentration of the fungicide at which mycelial growth was inhibited by $50 \%$. All values are means of two to four experiments, with three replicate petri dishes per experiment. Standard errors refer to the spiral gradient dilution assay.

b Values determined by Spiral Gradient Endpoint software with the 1- or the 2-day incubation option. 
after incubation at $25^{\circ} \mathrm{C}$ for 1 to 7 days, depending on the growth rate of the fungus.

In the conidial germination assays, conidial suspensions $(3 \times$ $10^{4}$ conidia per $\mathrm{ml}$ ) were prepared in sterile distilled water or (for P. digitatum) in a $0.01 \%$ solution of Triton X-100 (Sigma, St. Louis). Four 20- $\mu$ l droplets were evenly spaced on the agar surface in each petri dish, the droplets were allowed to dry in the airflow of a horizontal-flow transfer hood, and the plates were incubated overnight at $25^{\circ} \mathrm{C}$. In each droplet, 50 conidia were assessed microscopically for germination. A conidium was considered germinated if the length of the germ tube was at least twice the diameter of the conidium. Experiments using the agar dilution method were repeated at least once.

Calculation of $\mathbf{E C}_{50}$ values from agar dilution data. Fungal growth or germination data obtained by the agar dilution method were expressed as percent inhibition compared to growth on unamended agar, and data from repeated experiments were averaged. SAS (version 6.12, SAS Institute, Cary, NC) was used to regress probit-transformed inhibition data on the $\log _{10}$-transformed fungicide concentration. $\mathrm{EC}_{50}$ values for fungicide concentrations were obtained from the graphed regression line at the probit zero (50\% reduction) point.

Spiral gradient dilution method. Petri dishes $(15 \mathrm{~cm}$ in diameter) were placed on a level surface, and $50 \mathrm{ml}$ of PDA was poured into each dish, to form a layer of agar $3.3 \mathrm{~mm}$ thick, as suggested by the SGE manual. The plates were prepared at least $24 \mathrm{~h}$ before aqueous stock concentrations of fungicides were applied to the agar. Appropriate stock concentrations were determined by first evaluating several concentrations over a range, generally between 0 and 1,000 mg/liter (e.g., 10, 100, and 1,000 mg/liter). When data on the toxicity of a fungicide were available, the stock concentration could be more rapidly and accurately determined by the SGE software using the exponential mode calculations options.

A total of $50 \mu \mathrm{l}$ of a fungicide solution was spirally applied to each plate with a spiral plater (Spiral System Model D, Spiral Biotech) using the exponential mode of application, resulting in a radial gradient of approximately $1: 300$ in a $15-\mathrm{cm}$ dish. The start of the spiral was marked at the edge of the petri dish, and the center of the spiral was marked on the agar surface. The plates were incubated at room temperature for 2 to $4 \mathrm{~h}$ to allow the fungicide to diffuse and form a radial concentration gradient over the plate. A sterilized cork borer was used to remove an agar disk, approximately $25 \mathrm{~mm}$ in diameter, from the center of the plate. This region of the plate is fungicide-free, because the application of fungicide by the stylet of the spiral plater starts off-center. Removal of this agar disk prevented the growth of the fungus across the center of the plate and into neighboring inoculated areas.

Mycelium-covered cellophane strips or conidial suspensions were then radially applied across the fungicide concentration gradient on the spiral gradient dilution plate. A plate was placed on the template provided with the SGE software, with the start of the spiral at the No. 1 plate position. Forceps were used to carefully place cellophane strips over the plate position lines, with the mycelium facing down. Conidial suspensions $\left(5 \times 10^{5}\right.$ conidia per $\mathrm{ml}$ ) were applied in 10- $\mu$ l droplets on the periphery of the agar surface at the appropriate plate positions. The droplets were spread several times along the plate position lines with a sterile disposable plastic pellet pestle (Fisher Scientific, Pittsburgh), to evenly distribute them on the agar surface, without rupturing the surface. For each fungal isolate, a duplicate sample was placed at the opposite location on the plate, and three replicate plates were prepared for each fungicide. Depending on the growth rate of the fungus, up to four duplicated cellophane strips or eight duplicated streaks of a conidial suspension could be applied to a single plate. Controls consisted of PDA plates without fungicides, to which cellophane strips or spore suspensions were added. In the mycelial growth assays, the plates were incubated at $25^{\circ} \mathrm{C}$ for 1 day (R. stolonifer), 3 days (B. cinerea, C. gloeosporioides, and $M$. fructicola), or 4 or 5 days (C. purpureum, C. acutatum, Geotrichum citri-aurantii, and $P$. digitatum). In the conidial germination assays, the plates were incubated at $25^{\circ} \mathrm{C}$ for 14 to $20 \mathrm{~h}$.

Experiments using the spiral gradient dilution method were repeated at least once, and most of them were performed three or four times.

Calculation of $\mathbf{E C}_{50}$ values from spiral gradient dilution data. After incubation, the radial growth of the fungus in each of the three replicate control plates was measured (Fig. 1), and the values were averaged.

In the mycelial growth assays, the spiral gradient dilution plates were photographed to record the characteristic shape of the fungal growth. The location where fungal growth was inhibited by $50 \%$, compared with growth on the control plates, was determined as illustrated in Figure 1. The radial distance between this location and the center of the plate was measured. In the SGE software, this distance is a key parameter in determining the local fungicide concentration in the agar at the location of $50 \%$ inhibition.

Conidial germination was assessed microscopically. Plates were placed, bottom-side up, under the $10 \times$ objective of a light microscope (Axioskop, Zeiss, Jena, Germany). Conidia along the radial position lines on the spiral gradient plates were evaluated for germination. As in the agar dilution method, a conidium was considered germinated if the length of the germ tube was at least twice the widest diameter of the conidium. The location along the line where germination was inhibited by $50 \%$, compared with germination on the control plates, was first visually estimated and then more accurately determined by counting conidia. This location, which was generally a narrow zone, only a few millimeters wide, was marked on the petri dish. The distance between the $50 \%$ inhibition point and the center of the plate was then measured.

The following parameters were entered in the SGE software using the program option for single antimicrobial plates: plate size (10- or 15-cm petri dishes) and length of incubation after appli-

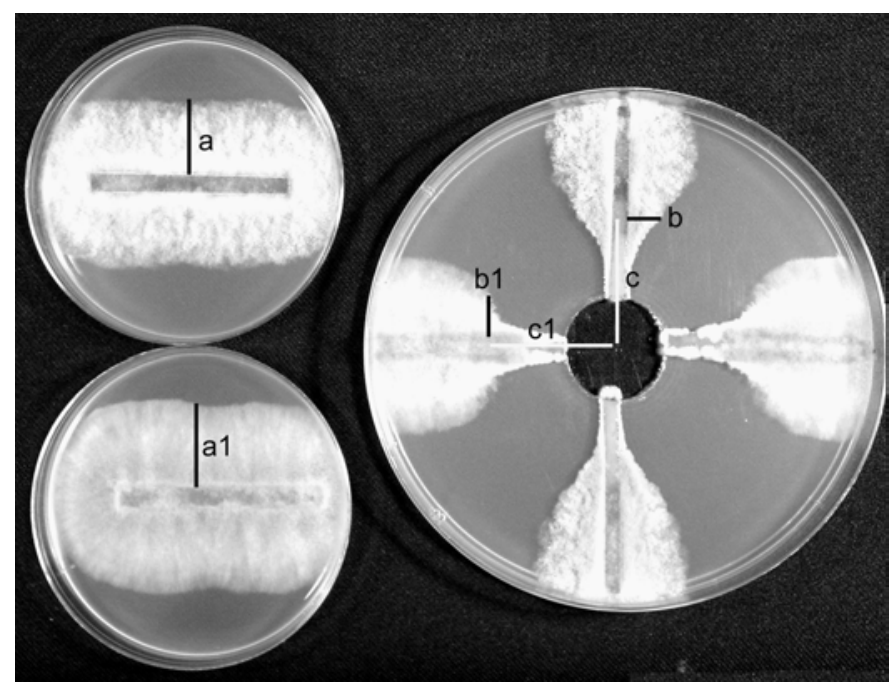

Fig. 1. Isolates of Botrytis cinerea and Monilinia fructicola grown on potato dextrose agar amended with dicloran (stock concentration, 2,000 mg/liter) applied in a spiral gradient dilution by means of a spiral plater, in a $15-\mathrm{cm}$ petri dish (right). Cellophane strips covered with mycelium of $B$. cinerea (left and right) and $M$. fructicola (top and bottom) were placed along radial lines across the fungicide concentration gradient. Controls for the two fungi were grown on unamended agar in $10-\mathrm{cm}$ petri dishes (M. fructicola, upper left; $B$. cinerea, lower left). After 3 days of incubation, three measurements were taken: radial growth of the controls (a and a1 for $M$. fructicola and $B$. cinerea, respectively), the location on the spiral gradient dilution plate where fungal growth is inhibited by $50 \%$ (b and b1 for $M$. fructicola and B. cinerea, respectively), and the distance between the center of the plate and the $50 \%$ growth point (c and c1 for $M$. fructicola and B. cinerea, respectively). 
cation of the fungus (1 or 2 days) for "plate-characteristics"; and fungicide used, fungicide stock concentration (in milligrams per liter) applied to the plate, and mode of application (exponential) for "antimicrobial". The molecular weights of the fungicides had been entered earlier into the antimicrobial database of the program, to calculate stock concentrations of the test substances. The radial distance (in millimeters) between the zone of $50 \%$ inhibition of growth or conidial germination and the center of the plate was entered as ER (ending radius) for each plate location between 1 and 15 in the "data entry area." The local fungicide concentration was then automatically displayed as EC (ending concentration). Additionally, a second local concentration can be obtained as TEC (tail ending concentration) by entering radial distances as TER (tail ending radius) for parameters such as the end of mycelial growth or outlier colonies in conidial streaks. Data sheets for each plate were then exported to a spreadsheet program (Excel, Microsoft Corp., Redmond, WA). Data for each fungus-fungicide combination were averaged for the duplicate fungal inocula on each plate, for the replicated petri dishes, and

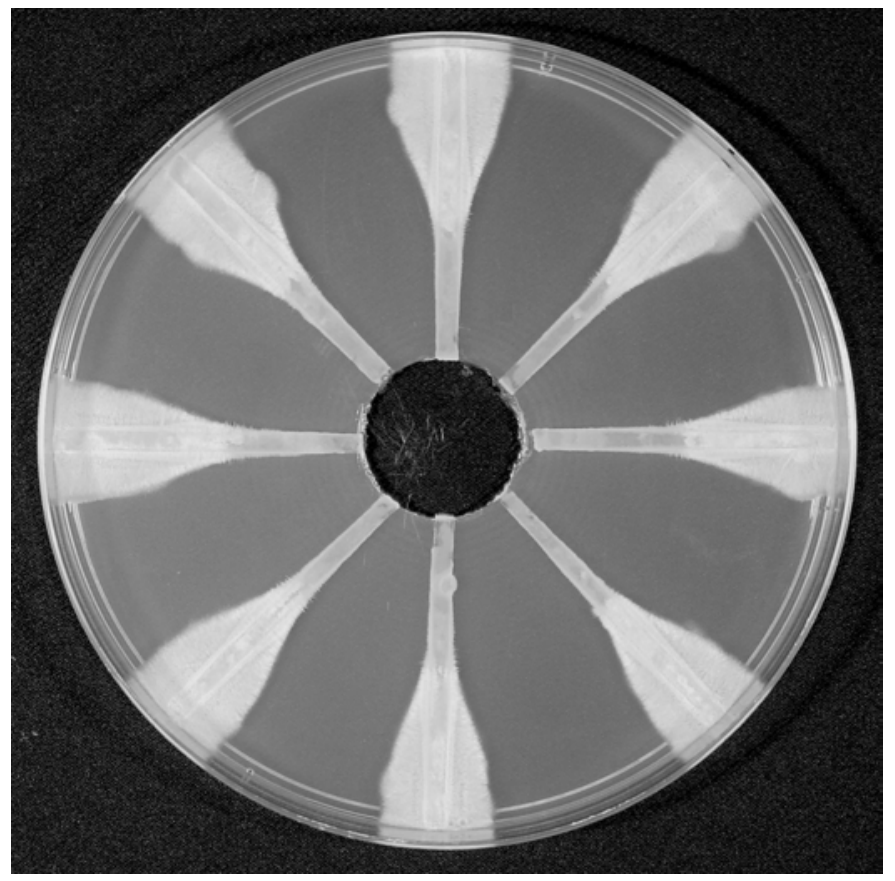

Fig. 2. Agar plate amended with tebuconazole (stock concentration, $5,000 \mathrm{mg} /$ liter) in a spiral gradient dilution and inoculated with cellophane strips covered with mycelium of four isolates of Geotrichum citri-aurantii, after 5 days of incubation. For each of the four isolates, a duplicate strip was placed in the opposite location on the plate. for the repeated experiments. Standard errors were calculated for repeated experiments.

Comparison of $\mathrm{EC}_{50}$ values obtained by the two methods. SigmaPlot (version 5.0, SPSS, Inc., Chicago) was used to graphically regress $\mathrm{EC}_{50}$ values (in milligrams per liter) obtained by the spiral gradient dilution method on values obtained by the agar dilution method. Regression lines were mathematically characterized for inhibition of mycelial growth and conidial germination, with either the 1-day or the 2-day option of the SGE program. SAS (version 8.2) was used to perform regression analyses on average $\mathrm{EC}_{50}$ values for all experiments with each fungus-fungicide combination. The slopes and $y$ intercepts of regression lines were compared, by means of $t$ tests (15), to a theoretical $1: 1$ relationship between the spiral gradient dilution method and the agar dilution method.

Estimated bias, coefficients of variation, and actual confidence intervals were also calculated for the mycelial and conidial assays. As a measure of accuracy, bias was defined as $100 \times(\beta-$ $1)$, where $\beta$ is the slope of the linear regression of the $\mathrm{EC}_{50}$ values of the two methods. Thus, bias estimates the systematic departure from a perfect 1:1 relationship with a slope of 1 . Positive and negative values indicate over- and underestimation, respectively. Coefficients of variation were calculated as $100 \times s_{\beta} / \beta$, where $s_{\beta}$ is the standard error of $\beta$. Actual confidence intervals were defined as $\hat{\beta} \pm s_{\hat{\beta}} t_{\mathrm{df} 0.05}$, where $\hat{\beta}$ is the predicted value of $\beta$ and $t_{\mathrm{df} 0.05}$ is the upper fifth percentile of a $t$ distribution with df degrees of freedom. Coefficients of variation and actual confidence intervals were used as estimates of precision.

\section{RESULTS}

Spiral gradient dilution method. Application of a fungicide solution to an agar plate by means of a spiral plater and incubation of the plate for 2 to $4 \mathrm{~h}$ resulted in a radial fungicide concentration gradient over the agar surface. As also visualized by the amount of fungal growth, the highest fungicide concentration was in the center and the lowest at the edge of the agar plate. A spiral gradient dilution plate prepared with dicloran and inoculated with isolates of $B$. cinerea and $M$. fructicola after 3 days of incubation is shown in Figure 1. Duplicates of the two isolates were applied on opposite sides of the plate. Control plates with unamended agar inoculated with the two fungi are shown for comparison. With slower-growing fungi, such as G. citri-aurantii, up to four isolates in duplicated strips could be evaluated on one plate (Fig. 2). Different fungal colony shapes were observed on the spiral gradient dilution plates with different fungus-fungicide combinations. Figure $3 \mathrm{~A}$ to $\mathrm{D}$ shows examples of plates inoculated with mycelium-covered cellophane strips. In the $C$. purpureum-myclobutanil interaction (Fig. 3A) and the C. acutatumtebuconazole interaction (Fig. 3B), both fungi grew over a wide
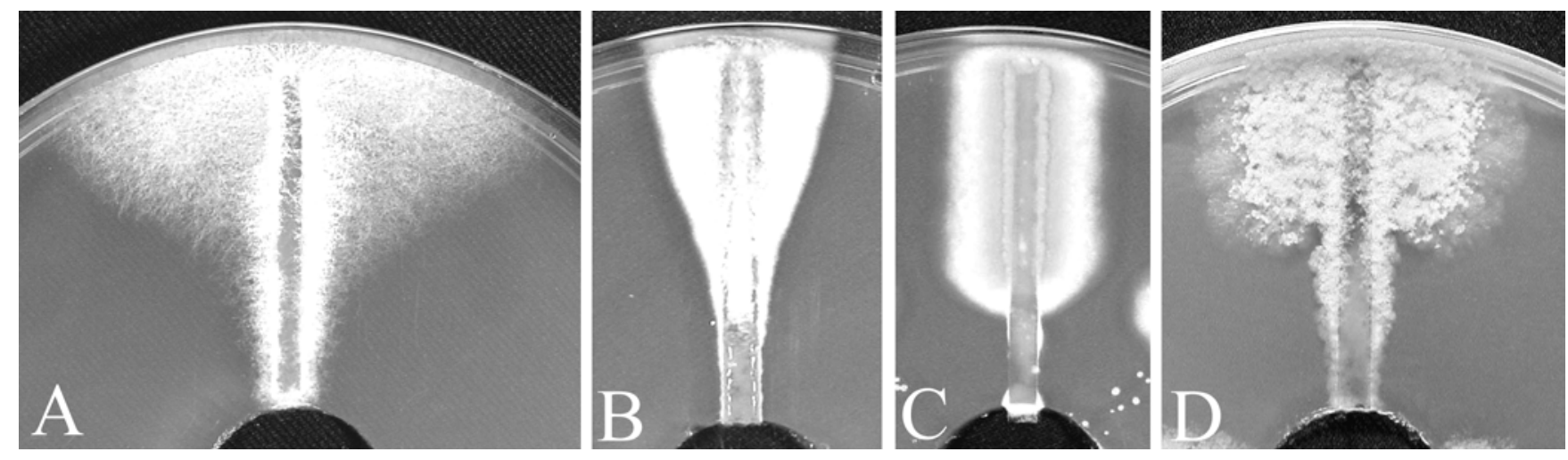

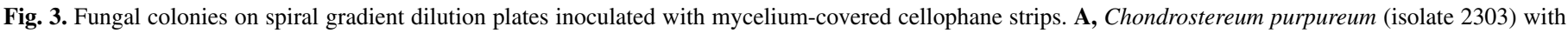

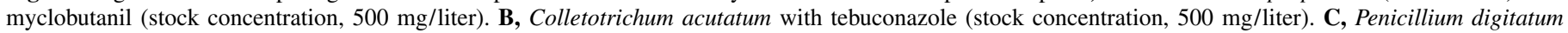
with iminoctadine tris-albesilate (stock concentration, $10 \mathrm{mg} /$ liter). D, Monilinia fructicola with fenhexamid (stock concentration, $30 \mathrm{mg} /$ liter). 
range of fungicide concentrations, and growth was gradually reduced with increasing concentration. In the $P$. digitatum-iminoctadine tris-albesilate interaction (Fig. 3C), there was a more abrupt transition from full growth to no growth of the fungus, within a narrow range of concentrations. The $M$. fructicola-fenhexamid interaction (Fig. 3D) was the most extreme, with almost no transition from full growth to very little growth of the fungus over a very narrow range of concentrations.

In the conidial germination assays, conidial streaks along the fungicide concentration gradient were microscopically evaluated for germination after 14 to $20 \mathrm{~h}$. When plates were incubated for 3 to 5 days, the colony shapes observed were similar to those in the mycelial growth tests. In addition, as shown in Figure 4 for the M. fructicola-myclobutanil interaction, other growth parameters can be characterized, such as the end of confluent growth (the beginning of the tail) and the colony outlier zone. In the example shown in Figure 4, the concentrations of myclobutanil at the end of confluent growth and at the end of the colony outlier zone of an isolate of $M$. fructicola was 0.38 and $0.68 \mathrm{mg} / \mathrm{liter}$, respectively, whereas the $\mathrm{EC}_{50}$ value for mycelial growth determined by this method was $0.15 \mathrm{mg} /$ liter. Outlier zones were also observed for $P$. digitatum with tebuconazole and tetraconazole and for B. cinerea and M. fructicola with fludioxonil.

Comparison of $\mathrm{EC}_{50}$ values obtained by the spiral gradient dilution and agar dilution methods. $\mathrm{EC}_{50}$ values for inhibition

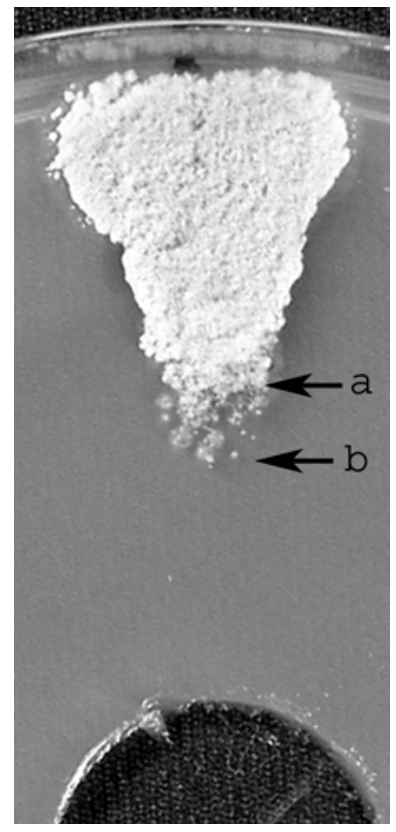

Fig. 4. Agar plate amended with myclobutanil (stock concentration, $500 \mathrm{mg} / \mathrm{liter}$ ) in a spiral gradient dilution and inoculated with a streak of a suspension of conidia of Monilinia fructicola, after 4 days of incubation. Growth characteristics indicated are a, the end of confluent growth (i.e., the beginning of the tail) and $\mathbf{b}$, the end of the colony outlier zone. of mycelial growth in 22 fungus-fungicide combinations determined by the spiral gradient dilution method are compared to values determined by the agar dilution method in Table 1. Except in four combinations ( $C$. acutatum-tebuconazole, $C$. acutatumpropiconazole, M. fructicola-tebuconazole, and P. digitatumtetraconazole), the values determined by the two methods were within a twofold dilution of one another. In the conidial germination assays, the two methods gave very similar results for all of the five fungicide-fungus combinations evaluated, with the 2-day incubation option in the SGE software (Table 2). With the 1-day option, all values were higher than those obtained with the 2-day option, but values obtained by the spiral gradient dilution method were still within one doubling dilution of values determined by the agar dilution method (Table 2). Thus, with an accuracy of one doubling concentration, there was $85.2 \%$ agreement between the two methods in determining inhibitory concentrations for mycelial growth and conidial germination in 27 fungus-fungicide interactions. In regression analyses for the data sets of $\mathrm{EC}_{50}$ values determined by the two methods, the models for the statistical analysis were highly significant in the mycelial growth assays $(P<0.0001)$ and the conidial germination assays $(P<0.01)$ with either the 1-day or the 2-day option (Fig. 5). Coefficients of determination $\left(r^{2}\right)$ were 0.93 or 0.92 in the mycelial growth assays and 0.94 or 0.97 in the conidial germination assays, with the 1-day or the 2-day option, respectively. The slope of the regression line was significantly different from 1 in the mycelial growth assays with the 1 -day option $(P<0.001)$ and in the conidial germination assays with the 2 -day option $(P<$ $0.035)$, indicating bias in the spiral gradient dilution method in these evaluations (Table 3). In the mycelial growth assays with the 2-day option $(P>0.080)$ and the conidial germination assays with the 1-day option $(P>0.664)$, however, the slope was not significantly different from 1 . In all cases, the $y$ intercept was not significantly different from 0 . The lowest estimated bias, or the highest accuracy, for comparing $\mathrm{EC}_{50}$ values was obtained with the 2-day option in the mycelial growth assays (bias $=13.5 \%$ ) and with the 1-day option in the conidial germination assays (bias $=7.5 \%$ ). For these assays and program options, low coefficients of variation and actual confidence intervals overlapping 1 in comparisons of regression slopes indicate that the method is of satisfactory precision (Table 3 ).

\section{DISCUSSION}

Results of this study demonstrate for the first time that a modification of the spiral gradient endpoint method, which was originally developed for determining minimum inhibitory concentrations of antimicrobial agents against bacteria, can be successfully used for determining $\mathrm{EC}_{50}$ values for fungicides. Because endpoints of fungal growth are not considered in this new application of the technique, we prefer the more general term spiral gradient dilution (SGD) method.

In our study, we evaluated 22 fungus-fungicide interactions for mycelial growth and five interactions for conidial germination.

TABLE 2. Values for $50 \%$ effective concentration $\left(\mathrm{EC}_{50}\right)$ for inhibition of conidial germination in selected fungus-fungicide combinations as determined by the agar dilution method and the spiral gradient dilution (SGD) method

\begin{tabular}{|c|c|c|c|c|c|c|c|}
\hline \multirow[b]{2}{*}{ Fungicide } & \multirow[b]{2}{*}{ Fungus } & \multirow[b]{2}{*}{ Isolate no. } & \multicolumn{5}{|c|}{$\mathrm{EC}_{50}$ for inhibition of conidial germination $(\mathrm{mg} / \mathrm{liter})^{\mathrm{a}}$} \\
\hline & & & Agar dilution & SGD 2-day ${ }^{\text {b }}$ & Std. error & SGD 1-day ${ }^{b}$ & Std. error \\
\hline \multirow[t]{2}{*}{ Fludioxonil } & Botrytis cinerea & 1356 & 0.070 & 0.061 & 0.001 & 0.104 & 0.002 \\
\hline & Monilinia fructicola & 90 & 0.040 & 0.046 & 0.004 & 0.076 & 0.005 \\
\hline Iminoctadine tris-albesilate & Penicillium digitatum & 1726 & 0.005 & 0.007 & 0.004 & 0.009 & 0.005 \\
\hline Tebuconazole & P. digitatum & 1726 & 0.055 & 0.061 & 0.008 & 0.098 & 0.013 \\
\hline Tetraconazole & $P$. digitatum & 1726 & 0.150 & 0.116 & 0.007 & 0.175 & 0.012 \\
\hline
\end{tabular}

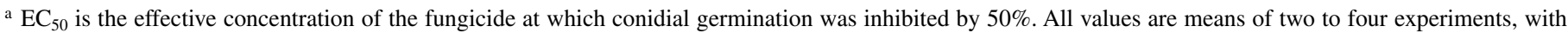

four replicate petri dishes per experiment. Standard errors refer to the spiral gradient dilution assay.

b Values determined by Spiral Gradient Endpoint software with the 1- or the 2-day incubation option. 
Only a small number of interactions were evaluated for conidial germination because the fungicides used are generally not good germination inhibitors; many conidia start germinating in the presence of these fungicides, but germ tube elongation and colony formation are subsequently inhibited. Because germ tube elongation is inhibited at different stages, evaluation of germination based on development of the germ tube to a length twice the

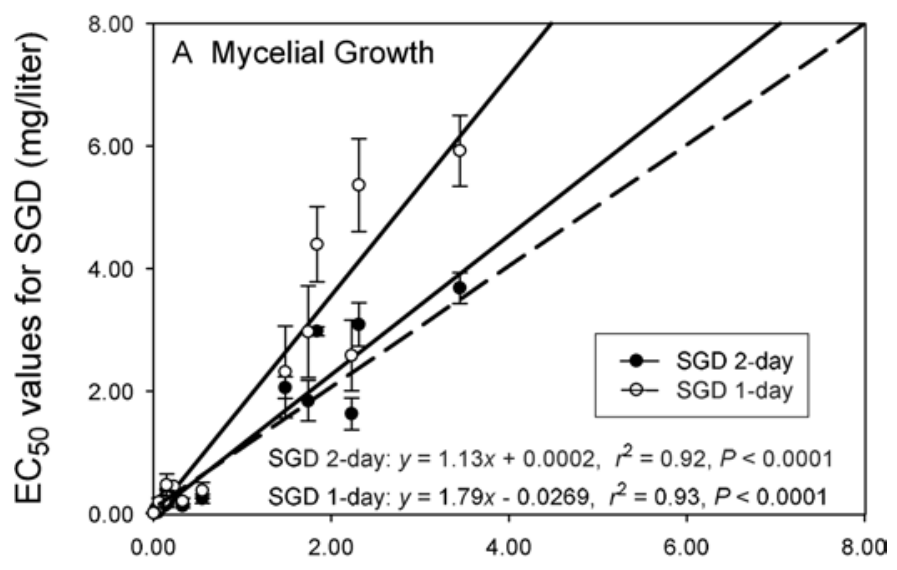

$\mathrm{EC}_{50}$ values for agar dilution method ( $\mathrm{mg} / \mathrm{liter}$ )

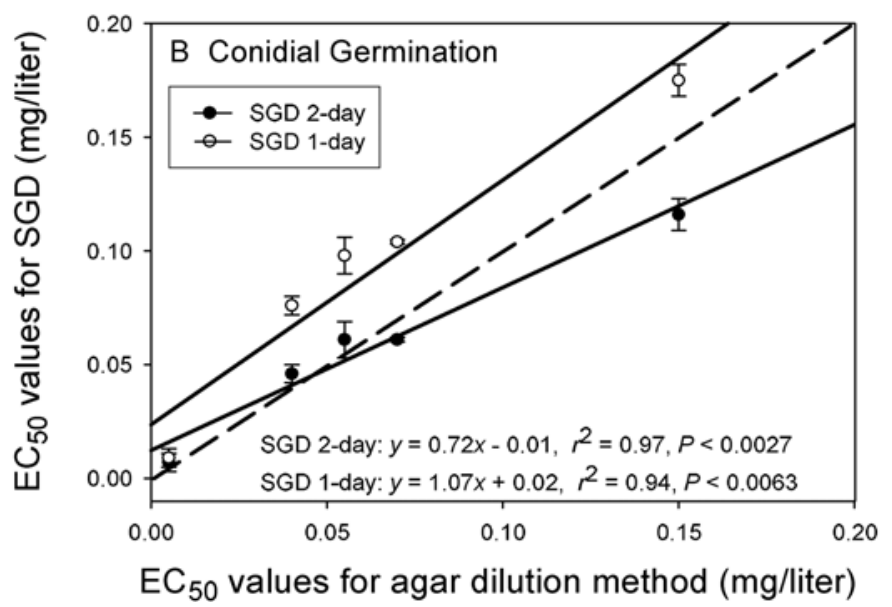

Fig. 5. Regression of $50 \%$ effective concentration $\left(\mathrm{EC}_{50}\right)$ values obtained by the spiral gradient dilution (SGD) method on those obtained by the agar dilution method for inhibition of $\mathbf{A}$, mycelial growth and $\mathbf{B}$, conidial germination. Data are shown in Tables 1 and 2. In the SGD method, $\mathrm{EC}_{50}$ values were determined by the Spiral Gradient Endpoint software using the 1- or 2-day incubation option. Data points are means of two to four experiments. The slope and $y$ intercept of the linear regression (solid lines) are compared with a 1:1 relationship (dashed lines). width of the conidium is often difficult. The fungi evaluated were members of the Zygomycota, Ascomycota, Basidiomycota, and Deuteromycota. The nine fungicides tested are members of six chemical classes and represent fungicides ranging from watersoluble (e.g., fenhexamid) to water-insoluble (e.g., benomyl).

Absolute accuracy cannot be claimed for any method that determines in vitro fungicide toxicity, but the traditional agar dilution method was used as a standard for comparisons. A high correlation was found between $\mathrm{EC}_{50}$ values for mycelial growth and conidial germination determined by the SGD method and values determined by the agar dilution method. In 23 of the total of 27 comparisons, the values determined by the two methods were within a twofold dilution of one another, resulting in $85.2 \%$ agreement between the two methods. Accuracy within a twofold dilution is the standard in bacterial testing (17). For fungi, however, a lower standard may be acceptable. Fungi often grow irregularly, with uneven margins that are difficult to trace and measure. Thus, errors can easily be introduced with any method used to assess $\mathrm{EC}_{50}$ values. Still, our statistical analyses validate the usefulness of the SGD method. In comparison to the agar dilution method, the accuracy of the SGD method was measured by the bias parameter. $\mathrm{EC}_{50}$ values were overestimated by only $13.5 \%$ in the mycelial growth assays and $7.5 \%$ in the conidial germination assays with the 2- and 1-day options of the SGE software, respectively. Mycelial agar plugs were used in the agar dilution method, whereas mycelium-covered cellophane strips were used in the SGD method. This difference in inoculum, the agar plug having a high nutrient content and the cellophane strip having a low nutrient content, could account for some of the variation in accuracy between the two methods, observed in regression analyses. Precision or repeatability was estimated by coefficients of variation and actual confidence intervals that included a slope of 1 , representing a 1:1 relationship between the SGD and agar dilution methods, in both the mycelial growth and the conidial germination assays. These analyses indicated that the SGD method was relatively both accurate and precise in comparison with the agar dilution method for calculating $\mathrm{EC}_{50}$ values of fungicides.

Other researchers have used either technical-grade fungicides (5) or formulated products $(8,19)$ for determining toxicity to fungi. When we determined $\mathrm{EC}_{50}$ values for fludioxonil, we obtained very similar values for technical-grade fludioxonil and for the formulated product Scholar 50WP, in tests for inhibition of $B$. cinerea and $M$. fructicola (data not shown). We utilized formulated materials for all our other comparisons because these products are being used in fungicide applications in the field. Adjuvants in formulated products can modify the activity of the fungicide or its diffusion rate in agar. Toxicity values, however, are never absolute, because even the agar medium may interfere with the activity of a fungicide (11).

TABLE 3. Statistical evaluation of the spiral gradient dilution method for estimating $50 \%$ effective concentration (EC ${ }_{50}$ ) of fungicides for inhibition of mycelial growth or conidial germination

\begin{tabular}{|c|c|c|c|c|c|c|c|c|c|}
\hline Assay & $\begin{array}{c}\text { Program } \\
\text { option }^{\mathrm{a}}\end{array}$ & $\begin{array}{l}\text { Number of } \\
\text { interactions }^{b}\end{array}$ & $\begin{array}{l}t \text { Value } \\
\text { slope }^{\mathrm{c}}\end{array}$ & $P$ value ${ }^{\mathrm{d}}$ & $\begin{array}{c}t \text { Value } \\
y \text { intercept }^{\mathrm{c}}\end{array}$ & $P$ value $^{\mathrm{d}}$ & $\begin{array}{l}\text { Estimated } \\
\operatorname{bias}^{\mathrm{e}}(\%)\end{array}$ & $\mathrm{CV}^{\mathrm{f}}(\%)$ & Actual CI ${ }^{g}$ \\
\hline Mycelial growth & $\begin{array}{l}\text { 1-day } \\
\text { 2-day }\end{array}$ & $\begin{array}{l}22 \\
22\end{array}$ & $\begin{array}{l}6.98 \\
1.84\end{array}$ & $\begin{array}{l}0.001 \\
0.080\end{array}$ & $\begin{array}{c}-0.197 \\
0.0022\end{array}$ & $\begin{array}{l}0.840 \\
0.999\end{array}$ & $\begin{array}{l}79.5 \\
13.5\end{array}$ & $\begin{array}{l}6.4 \\
6.5\end{array}$ & $\begin{array}{l}1.79 \pm 0.19 \\
1.14 \pm 0.14\end{array}$ \\
\hline Conidial germination & $\begin{array}{l}\text { 1-day } \\
2 \text {-day }\end{array}$ & $\begin{array}{l}5 \\
5\end{array}$ & $\begin{array}{l}0.48 \\
3.68\end{array}$ & $\begin{array}{l}0.664 \\
0.035\end{array}$ & $\begin{array}{l}1.89 \\
2.01\end{array}$ & $\begin{array}{l}0.155 \\
0.138\end{array}$ & $\begin{array}{r}7.5 \\
-28.5\end{array}$ & $\begin{array}{l}14.5 \\
10.8\end{array}$ & $\begin{array}{l}1.08 \pm 0.37 \\
0.72 \pm 0.18\end{array}$ \\
\hline
\end{tabular}

${ }^{a}$ Spiral Gradient Endpoint software option specifying incubation time before evaluation.

${ }^{\mathrm{b}}$ Number of fungus-fungicide combinations evaluated for mycelial growth or conidial germination.

${ }^{c}$ Comparison of the regression slope and $y$ intercept with a 1:1 relationship was done by $t$ tests.

d $P$ value is the lowest type 1 error from which to reject the null hypothesis that either the slope equals 1 or the intercept equals 0 .

${ }^{\mathrm{e}}$ Bias $=100 \times(\beta-1)$, where $\beta$ is the slope of the linear regression of the $\mathrm{EC}_{50}$ values determined by the spiral gradient dilution method as compared to values determined by the agar dilution method.

${ }^{\mathrm{f}}$ Coefficient of variation $(\mathrm{CV})=100 \times s_{\beta} / \beta$, where $s_{\beta}$ is the standard error of $\beta$.

g Confidence interval $(\mathrm{CI})=\left(\hat{\beta} \pm s_{\hat{\beta}} t_{\mathrm{df} 0.05}\right)$, where $s_{\hat{\beta}}$ is the standard error of the predicted value of $\beta(\hat{\beta})$ and $t_{\mathrm{df} 0.05}$ is the upper fifth percentile of a $t$ distribution with df degrees of freedom. 
Fungicide stock concentrations to be applied to the agar plate in the SGD method can be determined either empirically or by the SGE software if information on the toxicity of the fungicide is available. To determine local fungicide concentrations in the agar most accurately, a stock concentration should result in 50\% inhibition at locations in the midrange of the radial distance between the center and the periphery of the plate. Local fungicide concentrations along the concentration gradient are computed from several key parameters entered into the SGE program. The molecular weight of an antimicrobial agent, which is a major determinant of its diffusion rate, was incorporated into the computation formula with consideration of the opposing effects of radial and downward diffusion (2). Diffusion is also timedependent. Thus, after application of antimicrobial agents, plates should be incubated for 1 to $4 \mathrm{~h}$ to allow a gradient to form before the test organism is applied. After this period the gradient changes only very slowly. Still, a time factor for diffusion is included in the SGE software, with a 1- or a 2-day incubation option selected for determining $\mathrm{EC}_{50}$ values. We found that the optimal settings were the 2-day option in the mycelial growth assays and the 1day option in the conidial germination assays. We also noticed that the concentration gradients of the fungicides in our study were generally quite stable over the tested incubation time of up to 5 days. Thus, very similar $\mathrm{EC}_{50}$ values were obtained after 3, 4, and 5 days of incubation. Although $C$. purpureum is a relatively fast-growing fungus, its growth was evaluated after 5 days. Colony margins of this fungus were difficult to trace, and the longer incubation time minimized errors in the measurements. Mycelium-covered cellophane strips were applied as mycelial inoculum. Very similar $\mathrm{EC}_{50}$ values, however, were obtained by inoculation with agar strips containing mycelium in some fungus-fungicide interactions, i.e., C. purpureum-myclobutanil, $M$. fructicola-fludioxonil, and $M$. fructicola-tebuconazole (data not shown). The use of agar strips could further simplify the method, but this needs to be evaluated in more detail.

Fungal colony shapes varied in the different fungus-fungicide combinations studied. Shape characteristics were determined primarily by the fungicide but also by the fungus. The fungal colony shapes provide additional information on fungus-fungicide interactions. On a continuous fungicide concentration gradient, a fungus-fungicide interaction can be visualized as a dose-response growth curve. In general, fungal growth responded gradually to changes in fungicide concentrations. In some interactions, however, the response was more abrupt. For example, in the $M$. fructicola-fenhexamid interaction, there was almost no transition from full growth to very little growth of the fungus, over a very narrow range of fungicide concentrations. In this instance a better resolution of the fungicide concentration range might be obtained by using a spiral plater in the proportional mode of application, which is available in more advanced instruments and produces a narrower concentration gradient. Still, with a spiral plater in the exponential mode of application, concentrations of any fungicide can be obtained anywhere along the growth curve, and these values correlated well with values determined by the agar dilution method.

Gradient endpoints that characterize fungal growth and outlier colonies at the upper end of the concentration gradient may be important in additional applications of the technique, which we are currently investigating. Thus, for assessing the resistance potential of a fungicide in a pathogen population, fungal growth at the tail end of a conidial streak (located toward the center of the plate, where the fungicide concentration is highest) can be characterized (Fig. 4), and individual fungal colonies in the outlier zone can be isolated and their fungicide sensitivity can be studied in more detail. Furthermore, gradient endpoints for fungal growth might be used to determine minimum fungicide residue concentrations for specific pathosystems and thus could help to define usage rates for fungicides. Additionally, we found that the spiral plater, which was originally designed for the rapid enumera- tion of bacteria in suspension, was also useful for single-spore isolation of fungal isolates.

Because the spiral gradient endpoint method was developed for clinical bacteriology, the SGE software contains parameters and terms that are not commonly used in mycology and that are not needed for the application described here. Thus, not all data entry options in the program need to be filled in the current version. Radial distances defining the $50 \%$ inhibition point can be entered into either the ER (endpoint radius) box or the TER (tail-ending radius) box, and the local fungicide concentration will be displayed in the EC (ending concentration) box or the TEC (tailending concentration) box, respectively. Concentrations at any point along the growth curve can be determined, and these points can be given arbitrary names for characterizing a fungus-fungicide interaction. Other parameters in the program, such as GMIC (gradient minimum inhibitory concentration), were irrelevant to our application, because the traditional method of obtaining $\mathrm{EC}_{50}$ values uses regression analysis of inhibition data and is not done simply by a stepwise series of twofold agar dilutions, which is the method commonly used in bacterial studies.

In summary, the SGD method is a rapid, cost-effective, accurate, and reproducible (i.e., precise) alternative to traditional methods for quantification of the toxicity of fungicides. In conidial germination assays, a duplicate set of up to eight fungi can be grown on a single agar plate. In mycelial growth assays, a duplicate set of generally two to four fungi can be tested on a single plate. With a $2.5-\log$ dilution of a fungicide in a $15-\mathrm{cm}$ petri dish, a wide range of concentrations can be evaluated on a single plate. The more common $10-\mathrm{cm}$ petri dishes can also be used, resulting, however, in a narrower range of concentrations. The SGD method takes much less time than traditional methods and requires fewer supplies. The computer software for calculating fungicide concentrations is user-friendly and helpful in creating toxicity databases. At many institutions researchers have access to a spiral plater, and thus the only cost of setting up the technique in a laboratory is the purchase of the SGE software. In addition, cost savings will be considerable over time if many isolates are routinely tested. With its additional potential applications addressed above, the SGD method could become a valuable tool in fungus-fungicide studies.

\section{ACKNOWLEDGMENTS}

We thank H. Fukui for assistance in some of the agar dilution experiments and D. R. Jeske for helpful suggestions in our statistical analyses.

\section{LITERATURE CITED}

1. Atlas, R. M., and Parks, L. C. 1993. Microbiological Media. CRC Press, Boca Raton, FL.

2. Hill, G. B., and Schalkovsky, S. 1990. Development and evaluation of the spiral gradient endpoint method for susceptibility testing of anaerobic gram-negative bacilli. Rev. Infect. Dis. 12 (Suppl.):S200-S209.

3. Horsfall, J. G. 1945. Chapter III-Laboratory assay. Pages 14-41 in: Fungicides and Their Action. Chronica Botanica, Waltham, MA.

4. Horsfall, J. G. 1956. Chapter II-Fungicidal action and its measurement. Pages 15-29 in: Principles of Fungicidal Action. Chronica Botanica, Waltham, MA.

5. Köller, W., and Wilcox, W. F. 2001. Evidence for the predisposition of fungicide-resistant isolates of Venturia inaequalis to a preferential selection for resistance to other fungicides. Phytopathology 91:776-781.

6. Kuhajek, J. M., Jeffers, S. N., Slattery, M., and Wedge, D. E. 2003. A rapid microbioassay for discovery of novel fungicides for Phytophthora spp. Phytopathology 93:46-53.

7. Küng Färber, R. B., Chin, K. M., and Leadbitter, N. 2002. Sensitivity of Venturia inaequalis to trifloxystrobin. Pest Manage. Sci. 58:261-267.

8. Kunz, S., Deising, H., and Mendgen, K. 1997. Acquisition of resistance to sterol demethylation inhibitors by populations of Venturia inaequalis. Phytopathology 87:1272-1278.

9. Nakasone, K. K. 1990. Cultural Studies and Identification of WoodInhabiting Corticiaceae and Selected Hymenomycetes from North Amer- 
ica. Mycol. Mem. 15. J. Cramer, Berlin.

10. Olaya, G., and Köller, W. 1999. Baseline sensitivities of Venturia inaequalis populations to the strobilurin fungicide kresoxim-methyl. Plant Dis. $83: 274-278$

11. Osorio, J. M., Adaskaveg, J. E., and Ogawa, J. M. 1994. Inhibition of mycelial growth of Monilinia species and suppression and control of brown rot blossom blight of almond with iprodione and E-0858. Plant Dis. 78:712-716

12. Paton, J. H., Holt, H. A., and Bywater, M. J. 1990. Measurement of MICs of antibacterial agents by spiral gradient endpoint compared with conventional dilution method. Int. J. Exp. Clin. Chemother. 3:31-38.

13. Ribeiro, O. K. 1978. A Source Book of the Genus Phytophthora. J. Cramer, Vaduz, Liechtenstein.

14. Schaub, L., Sardy, S., and Capkun, G. 2002. Natural variation in baseline data: When do we call a new sample 'resistant'? Pest Manage. Sci. 58:959-963.

15. Scherm, H., and Copes, W. E. 1999. Evaluation of methods to detect fruit infected by Monilinia vaccinii-corymbosi in mechanically harvested rabbiteye blueberry. Plant Dis. 83:799-805.

16. Wexler, H. M., Molitoris, E., Jashnian, F., and Finegold, S. M. 1991. Comparison of spiral gradient and conventional agar dilution for susceptibility testing of anaerobic bacteria. Antimicrob. Agents Chemother. 35:1196-1202.

17. Wexler, H. M., Molitoris, E., Murray, P. R., Washington, J., Zabransky, R. J., Edelstein, P. H., and Finegold, S. M. 1996. Comparison of spiral gradient endpoint and agar dilution methods for susceptibility testing of anaerobic bacteria: A multilaboratory collaborative evaluation. J. Clin. Microbiol. 34:170-174.

18. Wong, F. P., and Wilcox, W. F. 2000. Distribution of baseline sensitivities to azoxystrobin among isolates of Plasmopara viticola. Plant Dis. 84: 275-281.

19. Yourman, L. F., Jeffers, S. N., and Dean, R. A. 2000. Genetic analysis of isolates of Botrytis cinerea sensitive and resistant to benzimidazole and dicarboximide fungicides. Phytopathology 90:851-859. 